\title{
Neurotrophins expression is decreased in lungs of human infants with congenital diaphragmatic hernia
}

This article was published in the following Dove Press journal:

Research and Reports in Neonatology

3 February 2014

Number of times this article has been viewed

Lynn D O'Hanlon

Sherry M Mabry

Ikechukwu I Ekekezie

Children's Mercy Hospitals/University of Missouri-Kansas City School of Medicine, Department of Pediatrics, Section of Neonatal-Perinatal Medicine, Kansas City, MO, USA
Correspondence: Lynn D O'Hanlon Neonatal Care PC, 707 N 190th Plaza, Omaha, NE 68022-3974, USA

Tel +l 4028 I5 I337

Fax + I 4028152055

Email lynn.ohanlon@hotmail.com
Objectives: To evaluate neurotrophin (NT) (nerve growth factor [NGF], NT-3, and brain-derived neurotrophic factor [BDNF]) expression in autopsy lung tissues of human congenital diaphragmatic hernia $(\mathrm{CDH})$ infants versus that of infants that expired with: 1$)$ "normal" lungs (controls); 2) chronic lung disease (CLD); and 3) pulmonary hypertension (PPHN).

Hypothesis: NT expression will be significantly altered in $\mathrm{CDH}$ lung tissue compared with normal lung tissue and other neonatal lung diseases.

Study design: Immunohistochemical studies for NT proteins NGF, BDNF, and NT-3 were applied to human autopsy neonatal lung tissue samples.

Subject selection: The samples included a control group of 18 samples ranging from 23-week gestational age to term, a CDH group of 15 samples, a PPHN group of six samples, and a CLD group of 12 samples.

Methodology: The tissue samples were studied, and four representative slide fields of alveoli/ saccules and four of bronchioles were recorded from each sample. These slide fields were then graded (from 0 to 3 ) by three blinded observers for intensity of staining.

Results: BDNF, NGF, and NT-3 immunostaining intensity scores were significantly decreased in the $\mathrm{CDH}$ lung tissue $(\mathrm{n}=15)$ compared with normal neonatal lung tissue $(\mathrm{n}=18)(P<0.001)$. The other neonatal pulmonary diseases that were studied, CLD and PPHN, were much less likely to be affected and were much more variable in their neurotrophin expression.

Conclusion: NT expression is decreased in CDH lungs. The decreased expression of NT in $\mathrm{CDH}$ lung tissue may suggest they contribute to the abnormality in this condition.

Keywords: nerve growth factor, NGF, brain-derived neurotrophic factor, BDNF, neurotrophin-3, NT-3, chronic lung disease, persistent pulmonary hypertension, lung development

\section{Introduction}

Neurotrophins (NTs) are a multigene family of polypeptide growth factors which include: nerve growth factor (NGF); brain-derived neurotrophic factor (BDNF); NT-3; NT-4/5; and NT-6. They exert their effects by binding to receptors on the surface of responsive cells. ${ }^{1-3}$ The physiologic role of NTs includes promoting differentiation and survival of developing neurons in the central and peripheral nervous system. ${ }^{1-4}$ Moreover, they have also been shown to stimulate differentiation and proliferation of other cell types. ${ }^{1,2,5-9}$

Congenital diaphragmatic hernia $(\mathrm{CDH})$ is a condition in which there is failed development of the diaphragm muscle during embryogenesis, along with pulmonary hypoplasia and pulmonary hypertension. ${ }^{10-13}$ Studies have shown that in CDH there is abnormal neural crest development including frequent abnormalities of the phrenic, 
vagus, and recurrent laryngeal nerves as a possible cause of incomplete development of the diaphragm and resulting hernia. ${ }^{14-16}$ However, it has also been shown in animal models of $\mathrm{CDH}$ that pulmonary hypoplasia precedes the occurrence of the diaphragmatic defect, suggesting an intrinsic deficiency in lung development is also present. ${ }^{17-19}$

Thus, existing data suggests that in $\mathrm{CDH}$ there may be a biomolecular defect which occurs early in development that interferes with nerve, diaphragm, and lung development prior to the visceral herniation through the diaphragm defect. If NT expression is abnormal or decreased, it may be a cause at the molecular level that would help to explain and unify all of these theories.

To begin to study this theory, we evaluated NT expression in autopsy lung tissues of human $\mathrm{CDH}$ infants versus that of infants that expired with: 1) "normal" lungs (controls); 2) chronic lung disease (CLD); and 3) pulmonary hypertension (PPHN). Here, we report that NT expression is decreased in the bronchial and parenchymal tissue of human $\mathrm{CDH}$ infant lungs.

\section{Materials and methods Tissue samples}

Autopsy lung specimens were selected from files (of the Department of Neonatology, Children's Mercy Hospital, Kansas City, MO, USA) based on infants who were histologically and clinically diagnosed with CDH, CLD, or PPHN. The control group included lung tissue from normal premature and term infants, 23-40 weeks gestational age (GA), who lived from 0 to 10 days, and died without lung disease. The lung tissue samples evaluated were obtained from a bank of previously collected (from 1985 to 2001), postmortem, paraffin-embedded, infant lung tissue blocks, of which we have previously published. ${ }^{20}$ Samples were obtained at autopsy, conducted within 8-24 hours after death. Lung tissues were perfused and fixed using a standard method. ${ }^{20}$ The control group included 18 samples from preterm to term gestation infants (23-40 weeks GA). The inclusion criteria for controls were that the premature infant lived $<48$ hours before significant pulmonary rearchitecturing had occurred, and the term infant died within 10 days after birth of non-pulmonary processes and required minimal ventilator support prior to death. The exclusion criteria included prolonged rupture of membranes ( $>48$ hours) and multiple congenital anomalies. The inclusion criteria for $\mathrm{CDH}(\mathrm{n}=15)$ samples were all specimens in which the infant had a clinical and postmortem diagnosis of CDH. These samples are from infants who lived for $<1$ to 49 days. Nine of these infants had bilateral- and the remainder had left diaphragmatic hernias. The samples used for this study were taken from the right lung or from the lobe which was least affected by hemorrhage. The CLD group consisted of 12 tissue samples from infants born from 23 to 29 weeks GA and had a clinical (oxygen dependency beyond 28 days of life or 36 weeks post conceptional age) and postmortem diagnosis of CLD. The inclusion criteria for the PPHN included six lung tissue samples from infants with the clinical and post mortem diagnosis of PPHN. Two of these infants were diagnosed with meconium aspiration syndrome, one with congenital anomalies, and three with primary PPHN. The exclusion criteria for all samples included extensive pulmonary hemorrhage seen histologically.

\section{Immunohistochemistry}

Formalin-fixed, paraffin-embedded lung tissue blocks cut into four micron sections were mounted on positively charged glass microscope slides and incubated at $60^{\circ} \mathrm{C}$. Slides were then selected in a blinded and random fashion from the four different groups (CDH, control, PPHN, and CLD), batched together for staining after deparaffinization and rehydration. Staining was enhanced by steaming slides in a target retrieval solution (DAKO, Carpentaria, CA, USA). Subsequently, the slides were incubated in blocking serum (DAKO). The primary antibody - rabbit immunoglobulin-G NT-3 (1:600), NGF (1:500), or BDNF (1:1500) (Santa Cruz Biotechnology, Inc, Santa Cruz, CA, USA) - was applied to the slide tissue sample and incubated. The slides were immunohistochemically stained by using a secondary antibody, and then they were treated with avidin-biotin-peroxidase complex (Vectastain ABC Elite kit, Vector Laboratories, Burlingame, CA, USA). The antigenic sites were visualized by the addition of the chromogen 3,3' diaminobenzidine and Harris hematoxylin (Fisher HealthCare, Houston, TX, USA). Negative control slides were stained using the same procedure, omitting the primary antibody.

\section{Analysis}

Each sample was evaluated under microscopy $(\times 400$ power) in a blinded/randomized fashion. For each sample, four fields per slide for both parenchyma and bronchioles were acquired with computerized image analysis software (AnalySIS Soft Imaging Systems, Lakewood, CO, USA). The staining intensity of the sections was scored by three blinded independent observers in a semiquantitative manner according to the following method: $0=$ no to scant staining, $1=$ light staining, $2=$ moderate staining, and $3=$ heavy staining (Figures 1 and 2). There were four fields per slide and three 


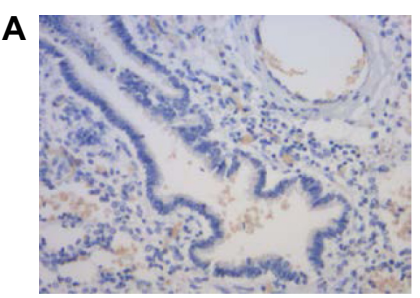

Score 0

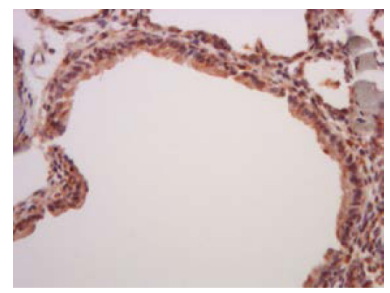

Score 2

B

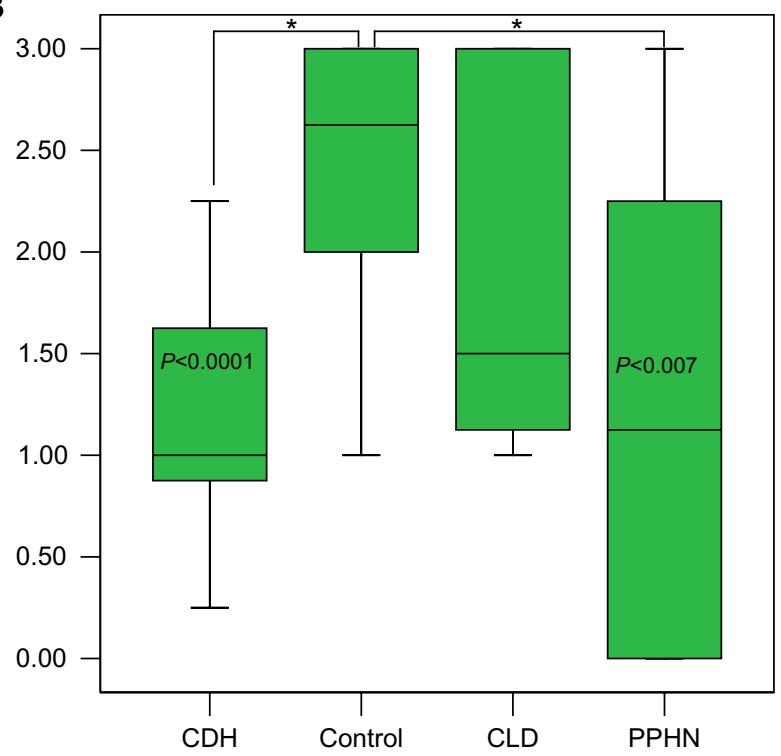

Figure I The NGF representative slide-staining $(\times 400)$ scores $(0-3)(\mathbf{A})$ and the corresponding intensity staining scores (B) at the bronchiole level.

Notes: The control group scores were: R I.00-3.00, IQR 2.00-3.00, and M 2.60 . $\mathrm{CDH}$ group scored significantly lower: $\mathrm{R} 0.25-2.25, \mathrm{IQR} 0.80-1.60, \mathrm{M}$ I.00, $P<0.000$ I, $\mathrm{Cl}$ 0.50-2.00. The CLD group scores versus control were not significantly different. The PPHN group appears significantly decreased: R 0.00-3.00, IQR 0.00-2.25, M I.I, $P<0.007, \mathrm{Cl} 0.30-2.20$, but the data are limited by the very wide range of scores and small sample size $(n=6)$. *Shows statistical significance as indicated.

Abbreviations: $\mathrm{CDH}$, congenital diaphragmatic hernia; $\mathrm{Cl}$, confidence interval; CLD, chronic lung disease; IQR, interquartile range; M, median; NGF, nerve growth factor; PPHN, pulmonary hypertension; $\mathrm{R}$, range.

observers; therefore, each slide was scored 12 times (at the bronchiole and parenchyma level). These scores were then averaged to give the final score of $0-3$ at 0.25 increments for the bronchiole and parenchymal staining intensity for each slide. SPSS 3.0 software (SPSS Inc., Chicago, IL, USA) was used to apply UNIANOVA (univariate analysis of variance) analysis to compare the control group to the diseased lung tissue groups. Each of the diseased lung tissue groups were
A

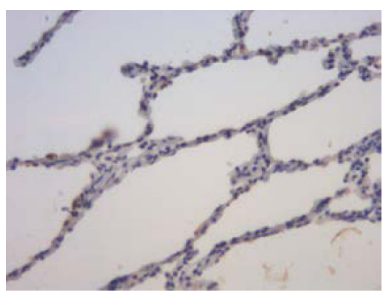

Score 0

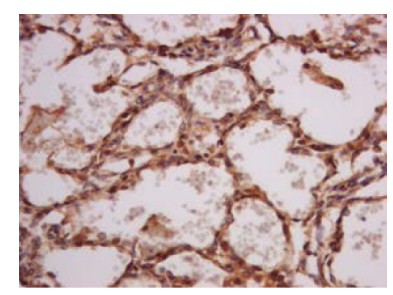

Score 2

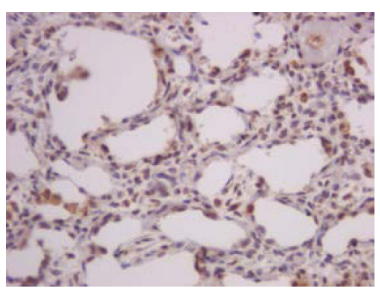

Score 1

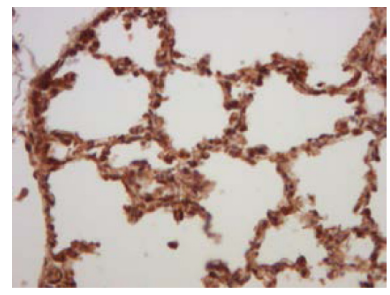

Score 3

B

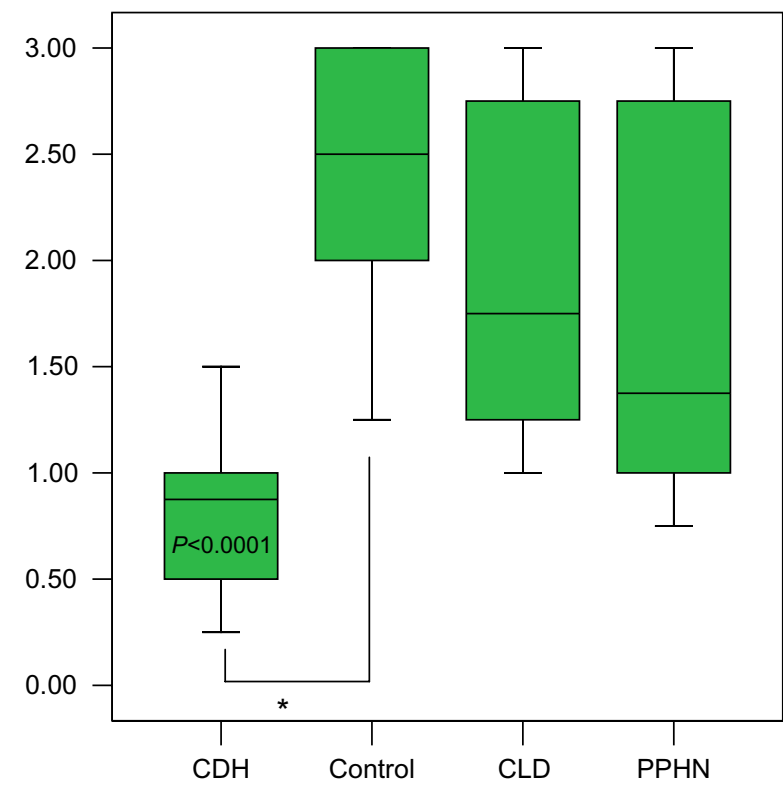

Figure 2 The NGF representative slide-staining $(\times 400)$ scores $(0-3)(\mathbf{A})$ and the corresponding intensity staining scores $(\mathbf{B})$ at the parenchymal level.

Notes: The control group scores were R I.25-3.00, IQR 2.00-3.00, M 2.50. The $\mathrm{CDH}$ group was significantly decreased, with scores: R $0.25-1.50$, IQR $0.50-1.00$, $M 0.90, P<0.000$ I, $C l$ I.00-2.30. The CLD and PPHN group scores were not significantly different versus control group. *Shows statistical significance as indicated. Abbreviations: $\mathrm{CDH}$, congenital diaphragmatic hernia; $\mathrm{Cl}$, confidence interval; CLD, chronic lung disease; IQR, interquartile range; $M$, median; NGF, nerve growth factor; PPHN, pulmonary hypertension; R, range.

then compared with one another by applying Tukey HSD (honestly significant difference) post hoc test analysis.

\section{Results}

The control lung tissue samples stained moderate to heavy for all three NTs. There were no significant differences in NT staining intensity at different GAs (23-40 weeks). For the CDH samples, all three NTs were significantly decreased at 
both the bronchiole and parenchyma levels, with NGF and NT-3 most impressively so. The CLD group was generally not significantly decreased and had a very wide range of staining intensity scores. The intensity of staining for the PPHN group appeared to be decreased, but with only six samples, the power is limited. For NGF staining at the bronchiole level (Figure 1A and B), control group scores range (R) 1.00-3.00, interquartile range (IQR) 2.00-3.00, with median (M) 2.60. The $\mathrm{CDH}$ group scored significantly lower: $\mathrm{R}$ 0.25-2.25, IQR 0.80-1.60, M 1.00, $P<0.0001$, confidence interval (CI) 0.50-2.00. The CLD group scores versus control were not significantly different. The PPHN group appears significantly decreased, R 0.00-3.00, IQR 0.00-2.25, M 1.1, P<0.007, CI $0.30-2.20$; however, the data are limited by very wide range of scores and small sample size $(n=6)$. NGF intensity scores at the parenchyma level (Figure $2 \mathrm{~A}$ and $\mathrm{B}$ ) for control were R 1.25-3.00, IQR 2.00-3.00, M 2.50. The CDH group was significantly decreased: R 0.25-1.50, IQR 0.50-1.00, M 0.90, $P<0.0001$, CI 1.00-2.30. The CLD and PPHN groups were not significantly different versus control group. For NT-3 at the bronchiole (Figure 3A and B) and parenchyma levels (Figure $4 \mathrm{~A}$ and $\mathrm{B}$ ), the $\mathrm{CDH}$ group had significantly less staining. At the bronchiole level (Figure $3 \mathrm{~A}$ and $\mathrm{B}$ ) scores were control group R 1.25-3.00, IQR 2.00-3.00, M 2.25 versus CDH group R 0.00-1.25, IQR 0.00-0.60, M 0.50, P<0.0001, CI 1.30-2.60. The CLD group had a very wide range of scores and was not significantly decreased versus control group. The PPHN group appeared significantly decreased: R 0.25-1.75, IQR 0.75-1.50, M 1.25, P<0.003, CI 0.30-2.00; but, as previously stated, had only six samples. At the parenchyma level (Figure 4A and B), the control group NT-3 intensity staining had scores R 1.25-3.00, IQR 2.00-2.50, M 2.1. The CDH group was again significantly decreased: R 0.00-1.00, IQR $0.50-1.00, \mathrm{M} 0.75, P<0.0001, \mathrm{CI} 0.90-2.10$. In this staining, the CLD group was significantly decreased relative to control. However, there was a wide range of scoring in this group: R 0.00-2.75, IQR 0.75-2.25, M 1.00, P<0.009, CI 0.20-1.50. The PPHN group was not statistically decreased. For BDNF, the intensity of staining for the $\mathrm{CDH}$ group was once again significantly decreased at both the bronchiole and parenchyma levels. At the bronchiole level (Figure 5A and B), the control group had intensity scoring R 1.00-3.00, IQR 1.75-3.00, M 2.25 versus the CDH group R 0.00-2.00, IQR 0.25-2.00, M 1.00, $P<0.001$, CI 0.40-1.90. Scores for CLD and PPHN groups were not significantly different versus control group. BDNF staining at the parenchyma level (Figure 6A and B) for the control group had scores R 0.75-3.00,
A
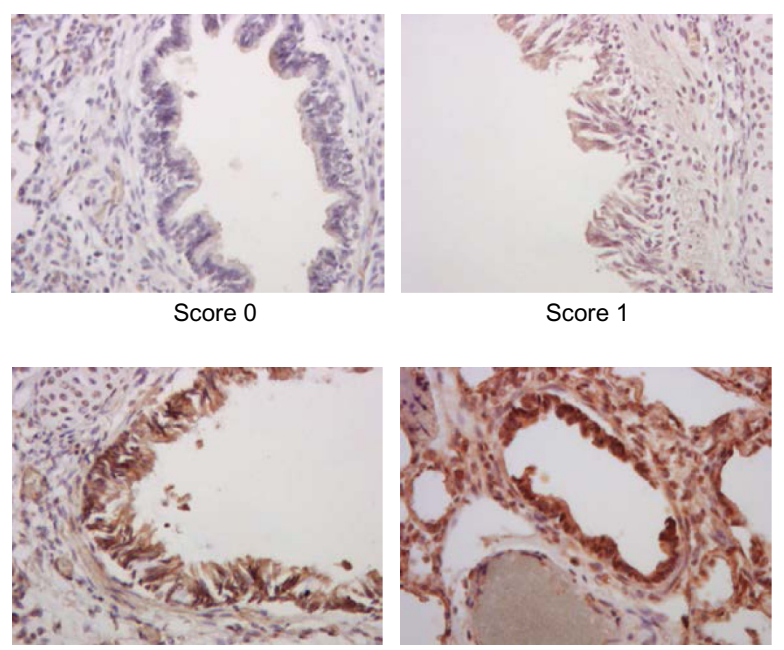

Score 2

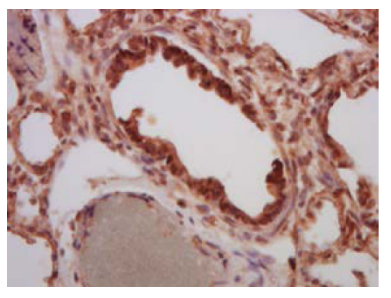

Score 3

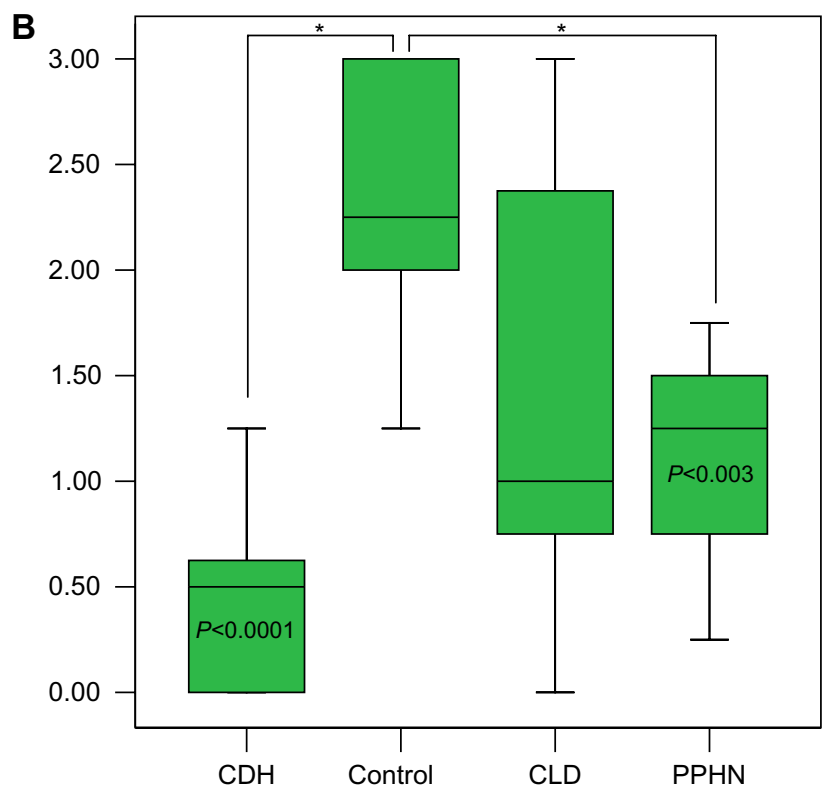

Figure 3 The NT-3 representative slide-staining $(\times 400)$ scores $(0-3)(\mathbf{A})$ and the corresponding intensity staining scores $(\mathbf{B})$ at the bronchiole level.

Notes: The control group scores were R I.25-3.00, IQR 2.00-3.00, M 2.25 versus $\mathrm{CDH}$ group $\mathrm{R} 0.00-1.25$, IQR $0.00-0.60, \mathrm{M} 0.50$. The $\mathrm{CDH}$ group had significantly lower scores, $P<0.000$ I, Cl I.30-2.60. The CLD group had a very wide range of scores and was not significantly decreased versus control group. The PPHN group appeared significantly decreased: R $0.25-1.75$, IQR $0.75-1.50$, M I.25, $P<0.003$, $\mathrm{Cl} 0.30-2.00$, but as previously stated, had only six samples. *Shows statistical significance as indicated.

Abbreviations: $\mathrm{CDH}$, congenital diaphragmatic hernia; $\mathrm{Cl}$, confidence interval; CLD, chronic lung disease; IQR, interquartile range; $M$, median; NT-3, neurotrophin 3; $\mathrm{PPHN}$, pulmonary hypertension; $\mathrm{R}$, range.

IQR 1.20-2.90, M 2.00. Relative to control, the CDH group was significantly decreased, with scores $\mathrm{R}$ 0.00-2.75, IQR 0.00-1.60, M 0.00; $P<0.004$, CI 0.30-2.00. The CLD and PPHN groups had a wide range of staining intensity scores, and were not significantly decreased from the control group scores. In the post hoc tests using Tukey HSD, CDH, 
A

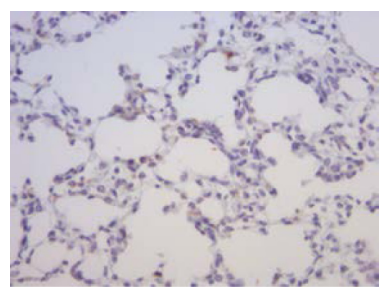

Score 0

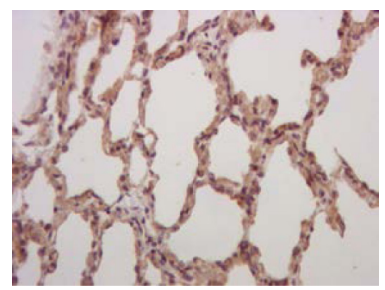

Score 2

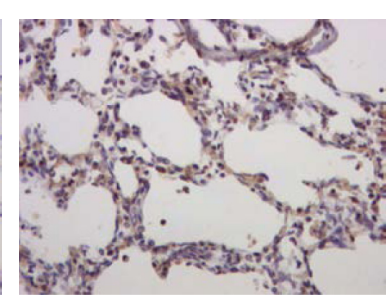

Score 1

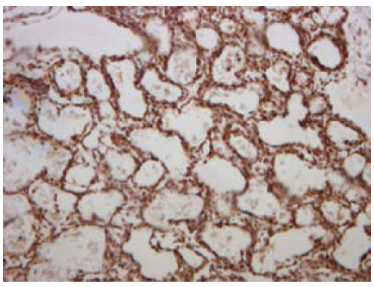

Score 3

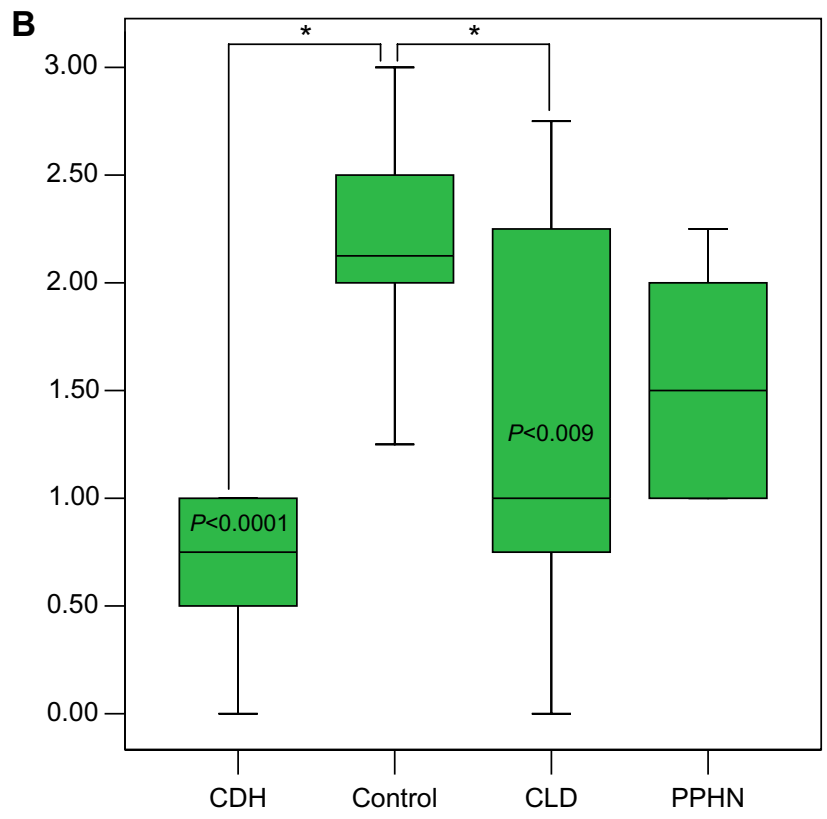

Figure 4 The NT-3 representative slide-staining $(\times 400)$ scores $(0-3)(\mathbf{A})$ and the corresponding intensity staining scores $(\mathbf{B})$ at the parenchymal level. The control group NT-3 staining intensity had scores R I.25-3.00, IQR 2.00-2.50, M 2.1, and the $\mathrm{CDH}$ group $\mathrm{R} 0.00-1.00$, IQR $0.50-1.00, \mathrm{M} 0.75$. The $\mathrm{CDH}$ group score is significantly lower versus control, $P<0.000 \mathrm{I}, \mathrm{Cl} 0.90-2.10$. In this staining, CLD group score was significantly decreased relative to control. However, there was a wide range of scoring in this group: $\mathrm{R} 0.00-2.75$, IQR $0.75-2.25, \mathrm{M} 1.00, P<0.009$, $\mathrm{Cl} 0.20-1.50$. The PPHN group score is not statistically decreased. *Shows statistical significance as indicated.

Abbreviations: $\mathrm{CDH}$, congenital diaphragmatic hernia; $\mathrm{Cl}$, confidence interval; CLD, chronic lung disease; IQR, interquartile range; $M$, median; NT-3, neurotrophin 3; $\mathrm{PPHN}$, pulmonary hypertension; $\mathrm{R}$, range.

CLD, and PPHN were compared with one another. In these analyses, $\mathrm{CDH}$ and PPHN were not statistically different from each other in staining intensity score for all NTs evaluated at both the bronchiole and parenchyma levels. $\mathrm{CDH}$ group score was significantly decreased compared with CLD at the bronchiole level for NGF staining intensity $(P=0.001$,

A

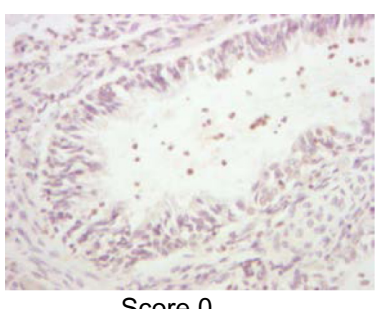

Score 0

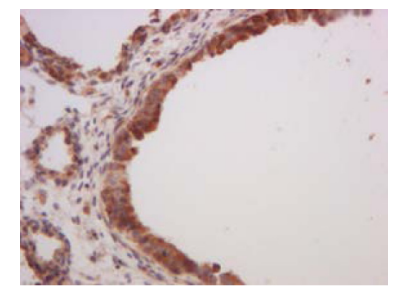

Score 2

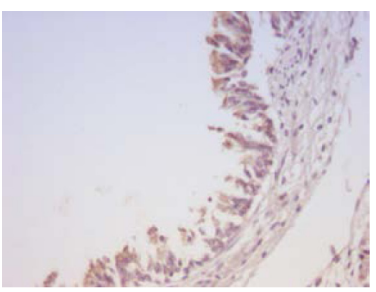

Score 1

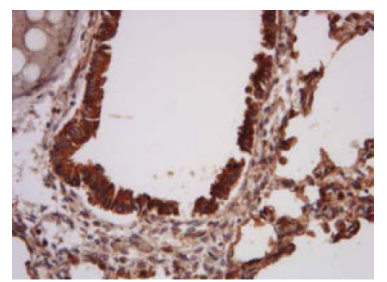

Score 3

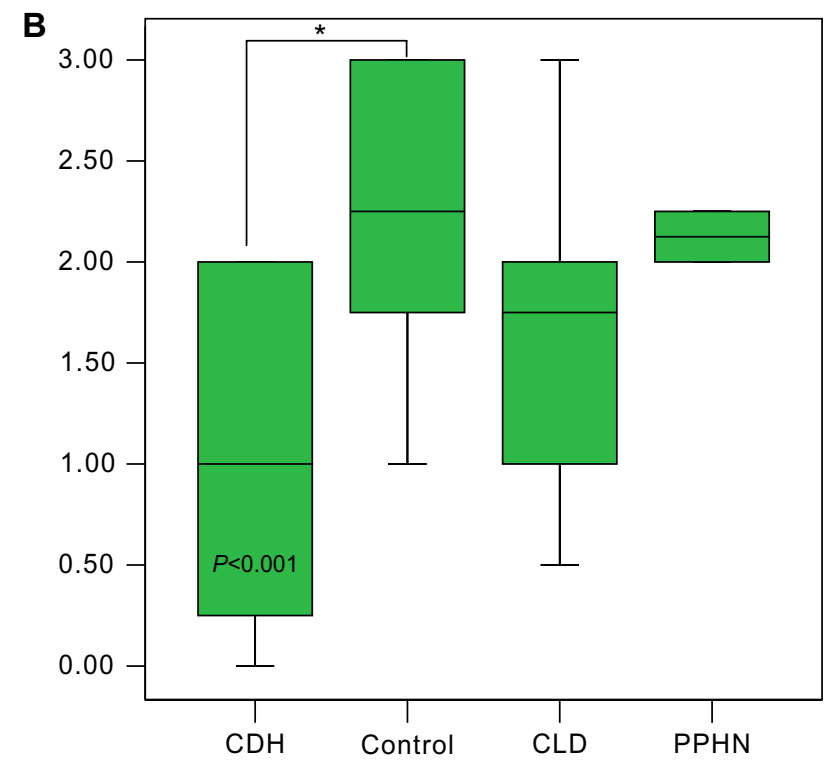

Figure 5 The BDNF representative slide staining $(\times 400)$ scores $(0-3)(\mathbf{A})$ and the corresponding intensity staining scores (B) at the bronchiole level. The control group had intensity scoring R I.00-3.00, IQR I.75-3.00, M 2.25, and the CDH group R 0.00-2.00, IQR 0.25-2.00, M I.00; CDH group has significantly lower scores, $P<0.001, \mathrm{Cl} 0.40-1.90$. Scores for CLD and PPHN groups were not significantly different versus control group. *Shows statistical significance as indicated.

Abbreviations: BDNF, brain-derived neurotrophic factor; $\mathrm{CDH}$, congenital diaphragmatic hernia; $\mathrm{Cl}$, confidence interval; $C L D$, chronic lung disease; $I Q R$, interquartile range; $M$, median; PPHN, pulmonary hypertension; $R$, range.

CI -1.90 to -0.40$)$ and significantly decreased at both the bronchiole $(P=0.002, \mathrm{CI}-1.80$ to -0.30$)$ and parenchyma $(P=0.05$, CI -1.40 to -0.01$)$ levels for NT-3. The staining intensity scores were significantly decreased for $\mathrm{CDH}$ compared with PPHN only at the parenchyma level for NT-3 ( $P=0.05$, CI -1.70 to -0.02$)$. 
A

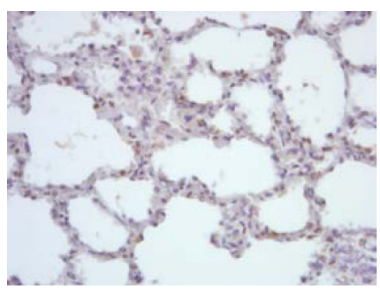

Score 0

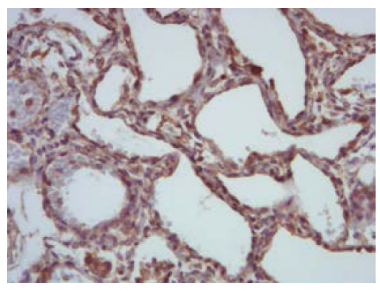

Score 2

\section{B}

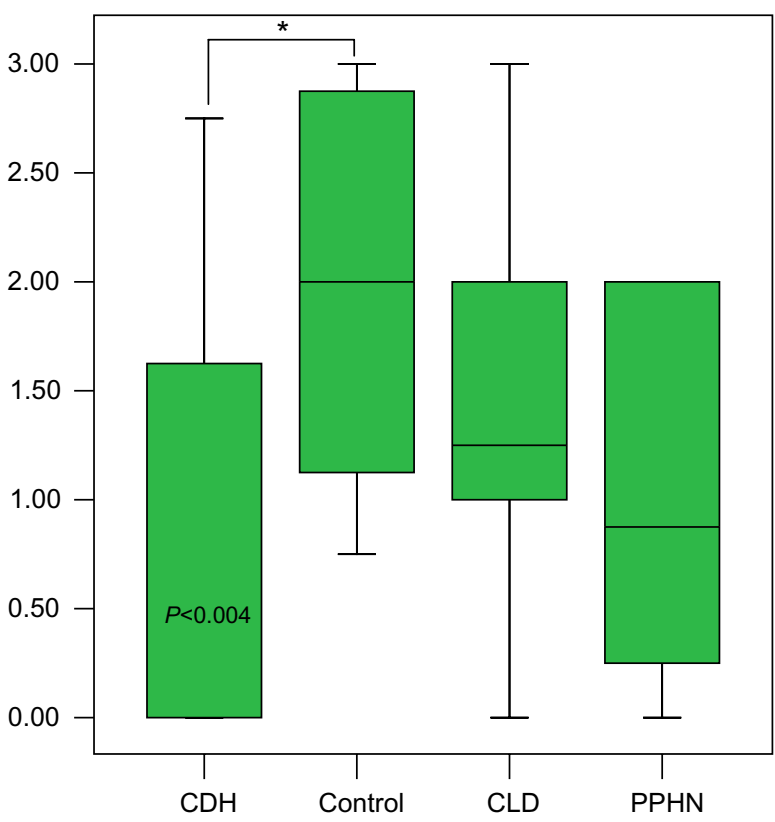

Figure 6 The BDNF representative slide staining $(\times 400)$ score $(0-3)(A)$ and the corresponding intensity staining scores $(\mathbf{B})$ at the parenchymal level. The control group had score R $0.75-3.00$, IQR I.20-2.90, M 2.00. Relative to control, the CDH group had significantly lower scores R $0.00-2.75$, IQR $0.00-1.60$, M $0.00 ; P<0.004$, $\mathrm{Cl} 0.30-2.00$. The CLD and PPHN groups had a wide range of staining intensity scores that were not significantly decreased versus control. *Shows statistical significance as indicated.

Abbreviations: BDNF, brain-derived neurotrophic factor; $\mathrm{CDH}$, congenital diaphragmatic hernia; $\mathrm{Cl}$, confidence interval; CLD, chronic lung disease; IQR, interquartile range; M, median; PPHN, pulmonary hypertension; $\mathrm{R}$, range.

\section{Discussion}

In the present study, we investigated the expression of NTs; specifically, NGF, NT-3, and BDNF, in human autopsy lung tissue of infants who died of congenital diaphragmatic hernia. We found significantly decreased expression of these NTs in the lung tissue of infants with $\mathrm{CDH}$ compared with lung tissue obtained from infants who died at 23-40 weeks GA without other common neonatal lung diseases. Furthermore, the NT expression in the $\mathrm{CDH}$ lung tissue samples was significantly decreased for all three NTs studied at both the bronchiole and parenchymal levels, which was unique to the $\mathrm{CDH}$ lung tissue compared with other common neonatal lung diseases that were studied. The expression of NTs in the CLD group of lung tissue was not significantly different than the control group, except in one group of samples: the NT-3 scores at the parenchyma level. However, in all groups of samples the scoring was quite variable. The PPHN samples tended to have decreased NT expression; however, this was statistically significant for only the NGF bronchiole tissue samples. With a limited sample size, it is hard to come to any conclusions. Furthermore, these samples were obtained from infants with PPHN secondary to a wide variety of underlying causes. The decreased expression of NTs seen in some of these samples may be related developmentally to $\mathrm{CDH}$. One is left to speculate that PPHN and CDH may be a spectrum of disturbance in the biochemical pathway that leads to decreased NT expression, and ultimately, disease. This is an area of research that requires more investigation. Although this study does not address whether the decreased NT expression is the underlying cause of $\mathrm{CDH}$, or simply the reflection of severity of illness, it is a first step in the investigations of the role of NTs in CDH. One can speculate that inflammation and steroid exposure, both known to affect NT expression, may be involved in the wide variability of expression seen in all of the autopsy specimens and that the NT expression was a secondary finding. ${ }^{1,18,21-24}$ This study was limited by the sample sizes, with a preponderance of bilateral as opposed to unilateral CDHs, and the use of autopsy specimens. Would live subjects with a wide variation in degree of illness, course of disease, and treatments show the same effects in NT expression? It is known that NTs are crucial for neural crest migration. ${ }^{1-9}$ Deficiency of NTs or their action is disruptive to proper development and maintenance of several organs, including the heart, and the cardiovascular and peripheral nervous system of the thoracic region. ${ }^{25}$ The disruption in the molecular biochemical pathway which results in $\mathrm{CDH}$ is not understood. Studies have shown abnormal tracheal and bronchopulmonary innervation in CDH. ${ }^{17-19}$ Other studies have shown the importance of NTs in lung development and neonatal lung diseases..$^{1-3}$ It is possible that the disruption or decreased expression of NTs in CDH plays an important role in this disease process. We have shown that there is an abnormality specific to lung tissue of $\mathrm{CDH}$ autopsy specimens, specifically decreased NT expression. Furthermore, this abnormality was seen with all NTs studied, which were 
unique to $\mathrm{CDH}$ compared with lung tissue of premature to term infants with or without other common neonatal lung diseases. We speculate that the decreased NT expression may be involved in the underlying pulmonary hypoplasia as well as the disruption in the diaphragm development. Further studies are needed to explore the role of deficient NT expression in the development of $\mathrm{CDH}$, specifically in the development of pulmonary hypoplasia and diaphragm dysgenesis.

\section{Acknowledgments}

The authors would like to thank Dr Stephen Simon (PhDStatistics), for help with statistical analysis, and Christine Concepción for her secretarial assistance in preparation of this manuscript. No grants, financial support, or support from manufacturers was received to complete this project.

\section{Disclosure}

The authors report no conflicts of interest in this work.

\section{References}

1. Prakash YS, Thompson MA, Meuchel L, et al. Neurotrophins in lung health and disease. Expert Rev Respir Med. 2010;4(3):395-411.

2. Yamamoto M, Sobue G, Yamamoto K, Terao S, Mitsuma T. Expression of mRNAs for neurotrophic factors (NGF, BDNF, NT-3, and GDNF) and their receptors (p75 NGFR, TrkA, TrkB, and TrkC) in the adult human peripheral nervous system and nonneural tissues. Neurochem Res. 1996;21:929-938.

3. Snider W. Function of the neurotrophins during nervous system development: what the knockouts are teaching us. Cell. 1994;77:627-638.

4. Maisonpierre P, Belluscio L, Friedman B, et al. NT-3, BDNF, and NGF in the developing rat nervous system: parallel as well as reciprocal patterns of expression. Neuron. 1990;5:501-509.

5. Ricci A, Felici L, Mariotta S, et al. Neurotrophin and neurotrophin receptor protein expression in the human lung. Am J Respir Cell Mol Biol. 2004;30:12-19.

6. Wheeler EF, Bothwell M. Spatiotemporal patterns of expression of NGF and the low-affinity NGF receptor in rat embryos suggest functional roles in tissue morphogenesis and myogenesis. J Neurosci. 1992;12(3): 930-945.

7. Tollet J, Everett A, Sparrow M. Development of neural tissue and airway smooth muscle in fetal mouse lung explants. Am J Respir Cell Mol Biol. 2002;26:420-429.

8. Sparrow M, Weichselbaum M, McCray P. Development of the innervation and airway smooth muscle in human fetal lung. Am J Respir Cell Mol Biol. 1999;20:550-560.
9. Tollet J, Everett A, Sparrow M. Spatial and temporal distribution of nerves, ganglia, and smooth muscle during the early pseudoglandular stage of fetal mouse lung development. Dev Dyn. 2001;221: $48-60$.

10. Harrison M, Adzick N, Nakayama D, DeLorimier A. Fetal diaphragmatic hernia: pathophysiology, natural history, and outcome. Clin Obstet Gynecol. 1986;29:490-501.

11. Allan D, Greer J. Pathogenesis of nitrofen-induced congenital diaphragmatic hernia in fetal rats. $J$ Appl Physiol. 1997;83:338-347.

12. Rottier R, Tibboel D. Fetal lung and diaphragm development in congenital diaphragmatic hernia. Semin Perinatol. 2005;29:86-93.

13. Greer JJ, Cote D, Allan DW. Structure of the primordial diaphragm and defects associated with nitrofen-induced CDH. J Appl Physiol. 2000;89:2123-2129.

14. Martinez L, Gonzalez-Reyes S, Burgos E, Tovar JA. The vagus and recurrent laryngeal nerves in experimental congenital diaphragmatic hernia. Pediatr Surg Int. 2004;20:253-257.

15. Pederiva F, Lopez RA, Rodriguez JI, Martinez L, Tovar JA. Bronchopulmonary innervation defects in infants and rats with congenital diaphragmatic hernia. J Pediatr Surg. 2010;45:360-365.

16. Lath NR, Galambos C, Rocha AB, Malek M, Gittes GK, Potoka DA. Defective pulmonary innervation and autonomic imbalance in congenital diaphragmatic hernia. Am J Physiol Lung Cell Mol Physiol. 2012; 302:L390-L398.

17. Guilbert TW, Gebb SA, Shannon JM. Lung hypoplasia in the nitrofen model of congenital diaphragmatic hernia occurs early in development. Am J Physio Lung Cell Mol Physiol. 2000;279:L1159-L1171.

18. Langwieler T, Fiegel HC, Alaamian M, et al. The relationship of diaphragmatic defect, liver growth, and lung hypoplasia in nitrofen-induced congenital diaphragmatic hernia in the rat. Pediatr Surg Int. 2004;20: 509-514.

19. Keijzer R, Liu J, Deimling J, Tibboel D, Post M. Dual-hit hypothesis explains pulmonary hypoplasia in the nitrofen model of congenital diaphragmatic hernia. Am J Pathol. 2000;156:1299-1306.

20. Thibeault DW, Mabry SM, Ekekezie II, Truog WE. Lung elastic tissue maturation and perturbations during the evolution of CLD. Pediatrics. 2000;106:1452-1459.

21. Yao Q, Zaidi SI, Haxhiu MA, Martin RJ. Neonatal lung and airway injury: a role for neurotrophins. Semin Perinatol. 2006;30:156-162.

22. Hoyle GW. Neurotrophins and lung disease. Cytokine Growth Factor Rev. 2003; 14:551-558.

23. Nockher WA, Renz H. Neurotrophins in inflammatory lung diseases: modulators of cell differentiation and neuroimmune interactions. Cytokine Growth Factor Rev. 2003;14:559-578.

24. Noga O, Hanf G, Schaper C, O'Connor A, Kunkel G. The influence of inhalative corticosteroids on circulating nerve growth factor, brainderived neurotrophic factor and neurotrophin-3 in allergic asthmatics. Clin Exp Allergy. 2001;31:1906-1912.

25. Cesca F, Yabe A, Spencer-Dene B, et al. Kidins220/ARMS is an essential modulator of cardiovascular and nervous system development. Cell Death Dis. 2011;2:e226.

\section{Publish your work in this journal}

Research and Reports in Neonatology is an international, peer-reviewed, open access journal publishing original research, reports, editorials, reviews and commentaries on neonatal health. The manuscript management system is completely online and includes a very quick and fair

\section{Dovepress}

peer-review system. Visit http://www.dovepress.com/testimonials.php to read real quotes from published authors. 